\title{
A remarkable recurrent nova in $M$ 31: The 2010 eruption recovered and evidence of a six-month period
}

\author{
M. Henze ${ }^{1}$, M. J. Darnley ${ }^{2}$, F. Kabashima ${ }^{3}$, K. Nishiyama ${ }^{3}$, K. Itagaki ${ }^{4}$, and X. Gao ${ }^{5}$ \\ ${ }^{1}$ Institut de Ciències de l'Espai (CSIC-IEEC), Campus UAB, C/Can Magrans s/n, 08193 Cerdanyola del Valles, Spain \\ e-mail: henze@ice.cat \\ 2 Astrophysics Research Institute, Liverpool John Moores University, IC2 Liverpool Science Park, Liverpool, L3 5RF, UK \\ e-mail: M. J.Darnley@l jmu.ac.uk \\ 3 Miyaki-Argenteus Observatory, Miyaki, 990-2492 Saga-ken, Japan \\ ${ }^{4}$ Itagaki Astronomical Observatory, Teppo, 990-2492 Yamagata, Japan \\ 5 Xingming Observatory, Mt. Nanshan, Urumqi, 830011 Xinjiang, PR China
}

Received 11 August 2015 / Accepted 9 September 2015

\section{ABSTRACT}

\begin{abstract}
The Andromeda Galaxy recurrent nova M31N 2008-12a has been caught in eruption nine times. Six observed eruptions in the seven years from 2008 to 2014 suggested a duty cycle of $\sim 1 \mathrm{yr}$, which makes this the most rapidly recurring system known and the leading single-degenerate Type Ia Supernova progenitor candidate; but no 2010 eruption has been found so far. Here we present evidence supporting the recovery of the 2010 eruption, based on archival images taken at and around the time of eruption. We detect the 2010 eruption in a pair of images at 2010 Nov. 20.52 UT, with a magnitude of $m_{R}=17.84 \pm 0.19$. The sequence of seven eruptions shows significant indications of a duty cycle slightly shorter than one year, which makes successive eruptions occur progressively earlier in the year. We compared three archival X-ray detections with the well-observed multi-wavelength light curve of the 2014 eruption to accurately constrain the time of their optical peaks. The results imply that M31N 2008-12a might actually have a recurrence period of $\sim 6$ months ( $175 \pm 11$ days), making it even more exceptional. If this is the case, then we predict that two eruptions per year will be observable soon. Furthermore, we predict that the next eruption will occur in late Sep. 2015. We encourage additional observations.
\end{abstract}

Key words. galaxies: individual: M 31 - novae, cataclysmic variables - stars: individual: M31N 2008-12a

\section{Introduction}

Classical and recurrent novae are a subclass of the cataclysmic variables that display luminous eruptions driven by a thermonuclear runaway on the surface of an accreting white dwarf (WD; see Bode \& Evans 2008; Bode 2010; Woudt \& Ribeiro 2014, for recent reviews). By definition, a recurrent nova (RN) is any classical nova $(\mathrm{CN})$ that has been observed in eruption more than once. Such a classification is limited by observational selection effects, certainly at the upper end, with observed recurrence times between 1-100 yr. Kato et al. (2014) predicted the lower limit of recurrence times to be $\sim 2$ months (for a $1.38 M_{\odot}$ WD with a mass accretion rate of $3.6 \times 10^{-7} M_{\odot} \mathrm{yr}^{-1}$ ).

Since the time of Edwin Hubble (1929), novae have been readily observed extragalactically, particularly in $\mathrm{M} 31$, which with an annual nova rate of $65_{-15}^{+16}$ eruptions (Darnley et al. 2006) and almost 1000 nova eruptions discovered to date (Pietsch et al. 2007 , see also the online database ${ }^{1}$ ) is the prime laboratory in which to study these systems. Shafter et al. (2015) has recently published an astrometric catalogue of $16 \mathrm{M} 31 \mathrm{RNe}$, whereas Williams et al. (2014, and in prep.) has released a catalogue of $11 \mathrm{M} 31$ nova progenitor systems. Both these studies indicate that the contribution of $\mathrm{RNe}$ to the overall nova population may be significantly larger than originally thought, in line with Galactic results from Pagnotta \& Schaefer (2014).

The remarkable recurrent nova M31N 2008-12a exhibits the shortest eruption duty cycle of any known nova, just $1 \mathrm{yr}$ (Darnley et al. 2014; Darnley et al. 2015a). Its short recurrence

\footnotetext{
1 http://www.mpe.mpg.de/ m31novae/opt/m31/index.php
}

time is driven by a high mass WD and high mass accretion rate (Henze et al. 2014, 2015; Kato et al. 2014, 2015). The first optical eruption was detected in 2008 Dec., with subsequent optical detections in 2009 Dec., 2011 Oct., 2012 Oct., 2013 Nov., and 2014 Oct. (see Table 1 for details). Serendipitous X-ray detections, later associated with earlier eruptions, were seen in 1992 Feb., 1993 Jan., and 2001 Sep. (Henze et al. 2014; Tang et al. 2014).

Initially, the "missing" 2010 eruption was not seen as troublesome until the full picture of the eruption history started to emerge after the 2013 eruption. At the time, only the 2008 eruption was known (the 2009 eruption was first announced by Tang et al. 2014) and the system had yet to be spectroscopically confirmed. With the growing wealth of eruptions and data, the hunt has been on to uncover evidence of an initially missed 2010 eruption. Based on, for example, data from the Palomar Transient Factory (Cao et al. 2012, see their Fig. 4), the largest windows of time in which the 2010 eruption could have occurred were Oct. 18-26 or Nov. 19-26.

In this Letter we present our recovery of the 2010 eruption of M31N 2008-12a. In Sect. 2 we describe the archival observations and their analysis. In Sect. 3 we present our results and discuss their impact on the recurrence time scale. We draw our conclusions in Sect. 4.

\section{Observations and data analysis}

We analysed archival optical data obtained with the following telescopes: (a) a Meade 200R $40 \mathrm{~cm} \mathrm{f} / 9.8$ reflector, plus 
Table 1. List of observed eruptions of M31N 2008-12a.

\begin{tabular}{|c|c|c|c|c|}
\hline $\begin{array}{l}t_{\max , \text { optical }^{a}} \\
\text { (UT) }\end{array}$ & $\begin{array}{l}t_{\max , \mathrm{X}-\mathrm{ray}}{ }^{b} \\
\text { (UT) }\end{array}$ & $\begin{array}{l}\text { Days since } \\
\text { last eruption }\end{array}$ & Source & References \\
\hline (1992 Jan. 29) & 1992 Feb. 05 & & X-ray (ROSAT) & 1 \\
\hline (1993 Jan. 04) & 1993 Jan. 11 & 341 & X-ray (ROSAT) & 1 \\
\hline (2001 Aug. 26) & 2001 Sep. 08 & & $\mathrm{X}$-ray (Chandra) & 2 \\
\hline 2008 Dec. 26 & & & Optical & 3 \\
\hline 2009 Dec. 03 & & 342 & Optical (Palomar Transient Factory) & 4 \\
\hline 2010 Nov. 20 & & 353 & Optical & 5 \\
\hline 2011 Oct. 23.49 & & 337.5 & Optical & $4,6,7,8$ \\
\hline 2012 Oct. 19.72 & $<2012$ Nov. 06.45 & 362.2 & Optical; X-ray (Swift) & $8,9,10,11$ \\
\hline 2013 Nov. 28.60 & 2013 Dec. $05.9 \pm 0.2$ & 405.1 & Optical (iPTF); X-ray (Swift) & $4,8,11,12,13$ \\
\hline 2014 Oct. $03.7 \pm 0.1$ & 2014 Oct. 13.6 & 309.1 & Optical (Liverpool Telescope); X-ray (Swift) & 8,14 \\
\hline
\end{tabular}

Notes. Updated version of Table 1 from Tang et al. (2014). ${ }^{(a)}$ Time of the optical peak, those in parentheses are extrapolated from the X-ray data (see Sect. 3.2). ${ }^{(b)}$ Time of the X-ray peak. ${ }^{(c)}$ Time since last eruption only quoted when consecutive detections occurred in consecutive years.

References. (1) White et al. (1995); (2) Williams et al. (2004); (3) Nishiyama \& Kabashima (2008); (4) Tang et al. (2014); (5) this Letter; (6) Korotkiy \& Elenin (2011); (7) Barsukova et al. (2011); (8) Darnley et al. (2015a); (9) Nishiyama \& Kabashima (2012); (10) Shafter et al. (2012); (11) Henze et al. (2014); (12) Tang et al. (2013); (13) Darnley et al. (2014); (14) Henze et al. (2015).

SBIG STL1001E camera, at Miyaki-Argenteus observatory, Japan (observers: F. Kabashima and K. Nishiyama); (b) a $50 \mathrm{~cm}$ f/6 telescope, with BITRAN BN-52E(KAF-1001E) camera, at Itagaki Astronomical Observatory, Japan (observer: K. Itagaki); and (c) a $35 \mathrm{~cm} \mathrm{f} / 6.9$ Celestron C14 Schmidt-Cassegrain telescope at Xingming observatory, China (observer: X. Gao). All observations were unfiltered and their dates are given in Table 2.

All images were reduced and calibrated in a homogeneous way, using the AstrOmatic ${ }^{2}$ software packages, SExtractor (Bertin \& Arnouts 1996) for source extraction, SWarp (Bertin et al. 2002) for image stacking, and SCAMP (Bertin 2006) for astrometric and photometric calibration. We optimised the image reduction procedures to correct for the background light of M 31 and specific detection thresholds were used to create clean source catalogues. The astrometric solutions were computed in SCAMP, using as a reference system the M 31 part of the Local Group Galaxy Survey (LGGS; Massey et al. 2006).

Aperture photometry was performed on all these images using SExtractor (v2.19.5); point-spread function (PSF) fitting was also performed by the Starlink photom package (v1.12-2; Disney $\&$ Wallace 1982) for comparative purposes. Photometric calibration was achieved using three LGGS stars common to all the images (stars \#12, \#14, and \#15, see Darnley et al. 2015a, their Table 2). Although these observations were all unfiltered, the data for the secondary standards is well matched to the $R$-band, with typical calibration accuracy $<2 \%$.

No source was detected at the position of M31N 2008-12a in eight of the ten observations (see Table 2). The faintest nearby resolved object in all of these images, J004528.55+415451.7 (star \#11, Darnley et al. 2015a), was detected with a S/N of $\sim 10$ in all images. This object has a similar brightness to the peak of M31N 2008-12a. The average photometry of this object yielded $m_{R}=17.880 \pm 0.049$, consistent with the LGGS value of $m_{R}=17.876$. In Table 2 we record the $3 \sigma$ limiting magnitudes of these eight observations. All ten observations were capable of detecting the nova at least 0.5 mag below peak light.

A source was found at the position of M31N 2008-12a in a pair of observations taken on 2010 Nov. 20. In the first observation, taken at Nov. 20.503 UT, a source was detected with $S / N=$ 4.2. Photometry of this source yielded $m_{R}=17.63 \pm 0.26$. In the second observation, 0.036 days later, the source appeared to

\footnotetext{
2 http://www.astromatic.net/
}

Table 2. Observation log.

\begin{tabular}{lll}
\hline \hline Date (UT) & Observatory & Photometry \\
\hline 2010 Nov. 11.548 & Miyaki-Argenteus & $>19.0$ \\
2010 Nov. 12.515 & Xingming & $>18.9$ \\
2010 Nov. 13.526 & Xingming & $>19.1$ \\
2010 Nov. 14.550 & Xingming & $>19.0$ \\
2010 Nov. 20.503 & Miyaki-Argenteus & $17.63 \pm 0.26$ \\
2010 Nov. 20.539 & Miyaki-Argenteus & $18.24 \pm 0.42$ \\
2010 Nov. 21.385 & Itagaki & $>18.8$ \\
2010 Nov. 24.517 & Miyaki-Argenteus & $>19.5$ \\
2010 Nov. 25.525 & Xingming & $>19.4$ \\
2010 Nov. 26.478 & Miyaki-Argenteus & $>19.8$ \\
\hline
\end{tabular}

have faded to $18.24 \pm 0.42(S / N=2.6)$. The stacking of these two observations gives $m_{R}=17.84 \pm 0.19(S / N=5.7)$.

In Fig. 1 we show the positional agreement between these detections and the 2012 eruption of M31N 2008-12a, discovered with the same telescope. The comparison figure (right panel) was created by inverting the 2012 image and overlaying it with the 2010 image, which had its white colour channel turned transparent. We also changed the colour cuts of the 2010 image to improve the contrast, resulting in the saturation of the sources. Residual deviations between the two WCS solutions were corrected by eye to have the neighbouring stars match as closely as possible. The figure clearly shows that the positions of the two detections are consistent to within $<1^{\prime \prime}$ (for 1'. 3 pixels). The remaining variance is common to most stars in the field and is dominated by alignment uncertainties and PSF distortions. Both positions are also consistent with the position of M31N 200812a obtained during the 2013 eruption: $\mathrm{RA}=0^{\mathrm{h}} 45^{\mathrm{m}} 28.81$, Dec $=$ +41 $54^{\prime}$ 9.' 9 (J2000; Darnley et al. 2014).

\section{Discussion}

\subsection{The 2010 eruption}

The missing 2010 eruption of M31N 2008-12a had been the one downside in the unfolding story of this remarkable recurrent nova. The 1992, 1993, and 2001 eruptions uncovered in archival X-ray observations by Henze et al. (2014) and Tang et al. (2014) strongly indicated that yearly eruptions had been ongoing for a 

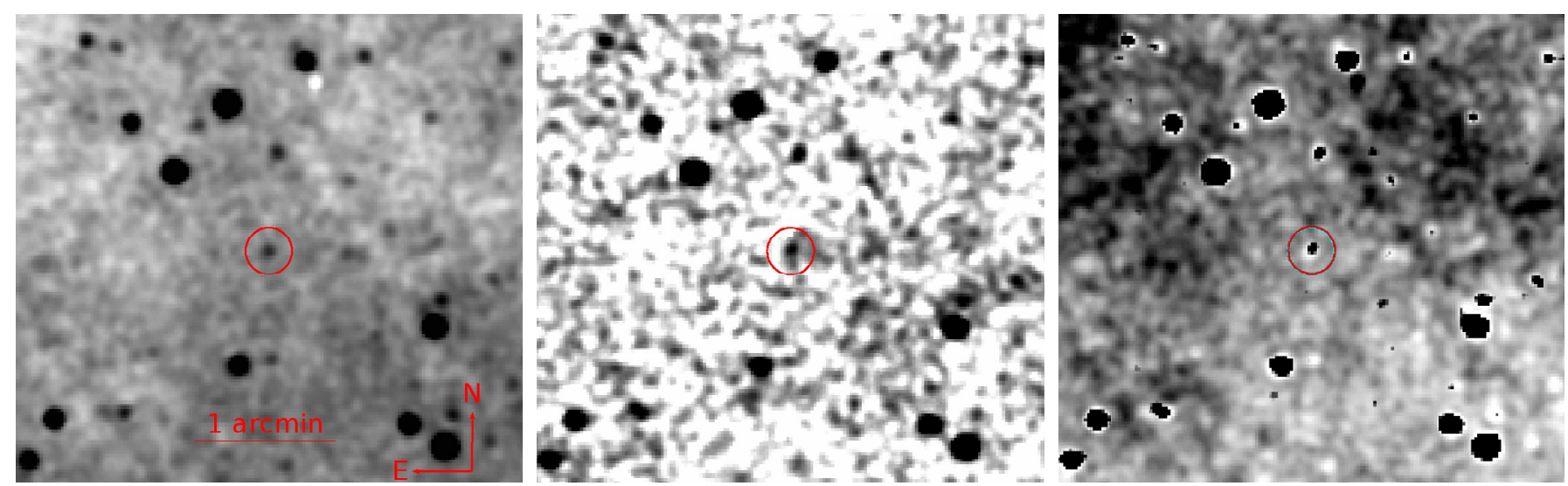

Fig. 1. Comparison of the M31N 2008-12a detections in 2010 and 2012. Left: stacked, smoothed image of the 2012 detection based on the MiyakiArgenteus data. Middle: stacked, background-subtracted, and smoothed image of the 2010 eruption combining the two detection images in Table 2. Right: astrometric overlay of the smoothed 2010 and 2012 stacks to show the positional coincidence. The 2012 image has been inverted and is in the background. The 2010 image has been saturated to enhance the contrast and its white channel turned transparent.

number of decades. The discovery of a possible vast remnant around the system by Darnley et al. (2015a) provides a tantalising hint at an eruption history over a significantly longer time scale.

The pair of clear detections of an object at the position of M31N 2008-12a allows us to constrain the 2010 eruption time of this system. The peak $R$-band detection of the 2014 eruption of M31N 2008-12a was at $m_{R}=18.2 \pm 0.1,0.038$ days after the peak luminosity, which is consistent with the $2010 \mathrm{de}-$ tection (within $2 \sigma$ ). Because of the unfiltered nature of these observations, there are likely to be some systematic uncertainties in these magnitudes, due in some part to the strong array of emission lines observed in the nova spectrum, and also to the spectral energy distribution which may peak beyond the nearUV (Darnley et al. 2015a). If we assume that the Nov 20.503 detection corresponds to maximum light then, using the later upper limits, we can constrain the decline time to be $t_{2} \leq 6$ days, consistent with the 2014 eruption of M31N 2008-12a $\left(t_{2}(R)=\right.$ $2.40 \pm 0.51$ days; Darnley et al. 2015a). The brightness of these detections, the positional coincidence, and the decline time limit, provide extremely compelling evidence that we have indeed recovered the elusive 2010 eruption of M31N 2008-12a.

By combining the eruption photometric data published in Darnley et al. (2014), Darnley et al. (2015a), we can update the template light curve of the eruption (Darnley et al. 2015a, see their Fig. 1). The updated $V$ - and $R$-band template light curves are shown in Fig. 2. While fairly unconstrained, this figure indicates that the 2010 detections fortuitously occurred extremely close to maximum light.

\subsection{Eruption statistics and evidence for a six-month period}

With a series of seven eruptions in seven years we can now begin to explore the statistics of the eruption time scales. The mean inter-eruption time is $351 \pm 13 \mathrm{~d}(0.962 \pm 0.036 \mathrm{yr})$. Here, the $1 \sigma$ uncertainty indicates the standard error of the mean. While this is still consistent with a $1 \mathrm{yr}$ recurrence period it suggests that the actual period could be slightly shorter. The distribution of eruption dates over time is shown in Fig. 3a. Here, we plot the days of the year of the optical peaks (compare Table 1). The fitted model suggests with $99 \%$ significance that successive eruptions are occurring at progressively earlier times during the year. Plotting the individual recurrence times over time does not reveal any trends.

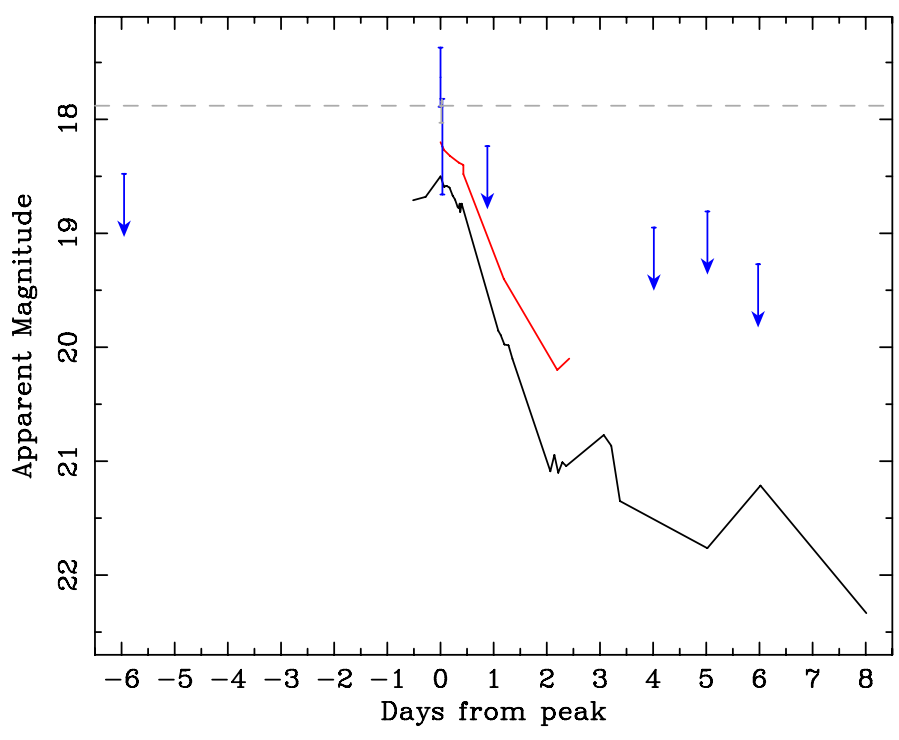

Fig. 2. M31N 2008-12a eruption template light curves based on $V$-band (black line) and $R$-band (red line) photometry from the 2008, 2011, 2012, 2013, and 2014 eruptions (Darnley et al. 2014, 2015a). The blue points indicate photometry of the 2010 eruption data (here we have assumed that the Nov. 20.503 observation occurred at maximum light), the arrow heads indicate the $3 \sigma$ upper limits from the non-detections, the tails the $5 \sigma$ limits. The grey dashed line indicates the reference photometry of the nearby star J004528.55+415451.7.

To further investigate the possibility of a recurrence time slightly below $1 \mathrm{yr}$ we revisited the historical X-ray detections listed in Table 1. Both ROSAT detections (in 1992/3) have good coverage of the emerging X-ray emission (White et al. 1995). Therefore, we could use the carefully observed 2014 X-ray light curve (Henze et al. 2015) as a template to extrapolate the dates of the optical peak with an accuracy of less than a day. The 2001 eruption was only detected in a single Chandra observation (Williams et al. 2004). However, the short duration of the $\mathrm{X}$-ray phase allowed us to constrain the optical peak to within two weeks, which is the size of the symbols in Fig. 3b.

In Fig. $3 b$ we add the resulting dates to the optical data. The two early dates were counted from the start of the previous year. There is little agreement with the trend found in Fig. 3a. This could of course indicate that no trend exists and that the random 

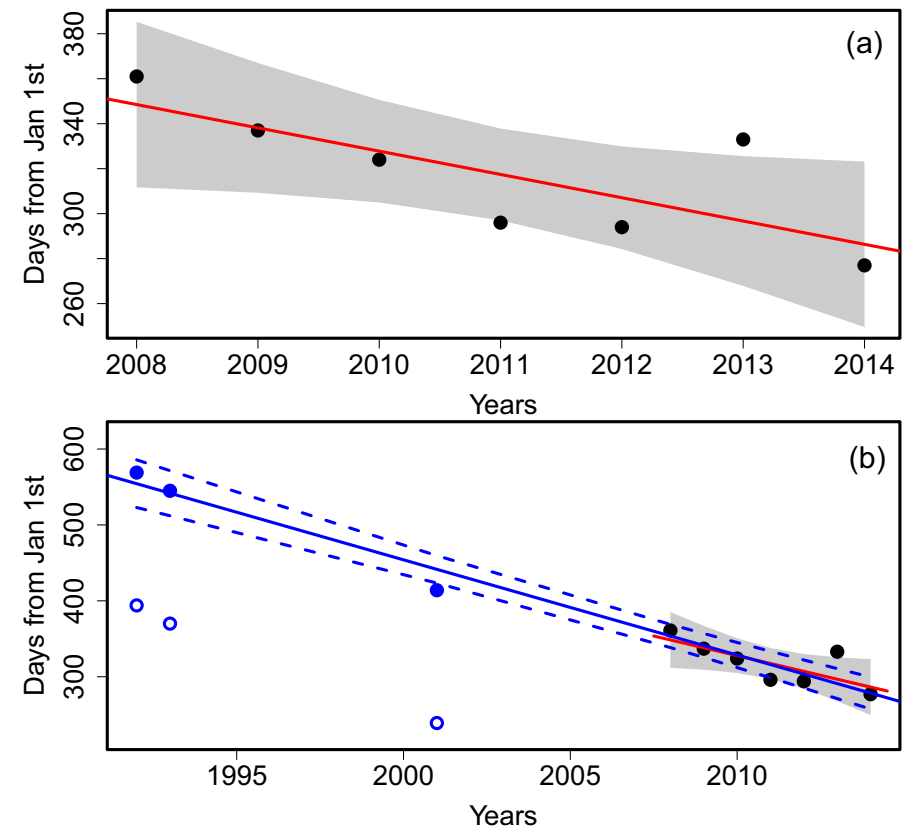

Fig. 3. Distribution of eruption dates (in days of the year) over time, based on Table 1, for the optical detections only (panel a)) and for all detections (panel b)). The red line is the best fit to the optical data (in black). The grey area is the corresponding 95\% confidence region. In panel b) the blue open circles are the optical peak dates corresponding to the X-ray detections (see the text). The blue filled circles are the same dates shifted by $175 \mathrm{~d}$. The blue solid line is the best fit to all solid data points and the blue dashed lines mark its $95 \%$ confidence region.

variance is high. However, if the three eruption dates are shifted upward by 175 days, which is half the apparent eruption period, there is excellent agreement with the trend from Fig. 3a. The overall fit is significant at the $5 \sigma$ level.

This finding is the first evidence that M31N 2008-12a might in fact have a recurrence period of $\sim 6$ months. During the last seven years, the first eruption of each year would have occurred while M 31 was too close to the sun to be observable. This situation is changing, as shown by the progressively earlier second eruptions. If the period really is $\sim 6$ months, then two eruptions per year should become observable soon: the overall fit predicts that the first eruption in 2017 will occur around early March, with $1 \sigma$ uncertainties of $24 \mathrm{~d}$. Our ongoing quiescent monitoring of the system will test this prediction. It also allows us to confidently rule out even shorter periods.

We estimate the actual recurrence period to be $175 \pm 11 \mathrm{~d}$ $(0.48 \pm 0.03 \mathrm{yr})$. There is no evidence that this period has changed over the last $\sim 20$ yr. The estimate takes into account the time between the 1992/3 eruptions and is consistent with the linear fit in Fig. 3b. Based on this fit we predict that the next observable eruption will occur in late September 2015, with a $1 \sigma$ prediction range from early September to mid October. We encourage additional observations.

\section{Conclusions}

In this Letter we have outlined the recovery of the elusive 2010 eruption of M31N 2008-12a and its impact on our knowledge of this exceptional object. Here we summarise our findings.

1. We have uncovered the previously missing 2010 eruption of the remarkable recurrent nova M31N 2008-12a from a pair of observations taken at 2010 Nov. 20.52 UT. The photometry of the nova at this time $m=17.84 \pm 0.19$ is consistent with the nova being at or around maximum light.

2. The sequence of seven eruptions in seven years strongly suggests that eruptions occur progressively earlier every year. We revisited archival X-ray data and found the resulting eruption dates to be consistent with this picture

3. The dates of the X-ray detections provide the first evidence that M31N 2008-12a might have two eruptions per year. The corresponding average recurrence period is $175 \pm 11 \mathrm{~d}(0.48 \pm$ $0.03 \mathrm{yr}$ ) and shows no evidence of change over a $\sim 20 \mathrm{yr}$ time span. In this scenario, two eruptions per year should become observable soon. We predict that the next eruption will occur in late Sep. 2015.

Note added in proof. While the paper was with the referee, the 2015 eruption was discovered on Aug. 28 (see Darnley et al. 2015b), slightly ahead of the prediction but entirely consistent with Fig. 3.

Acknowledgements. We are grateful to an anonymous referee for encouraging feedback. M.H. acknowledges the support of the Spanish Ministry of Economy and Competitiveness (MINECO) under the grant FDPI-2013-16933.

\section{References}

Barsukova, E., Fabrika, S., Hornoch, K., et al. 2011, ATel, 3725

Bertin, E. 2006, in Astronomical Data Analysis Software and Systems XV, eds. C. Gabriel, C. Arviset, D. Ponz, \& S. Enrique, ASP Conf. Ser., 351, 112 Bertin, E. \& Arnouts, S. 1996, A\&AS, 117, 393

Bertin, E., Mellier, Y., Radovich, M., et al. 2002, in Astronomical Data Analysis Software and Systems XI, eds. D. A. Bohlender, D. Durand, \& T. H. Handley, ASP Conf. Ser., 281, 228

Bode, M. F. 2010, Astron. Nachr., 331, 160

Bode, M. F., \& Evans, A. 2008, Classical Novae, 2nd edn. (Cambridge University Press), Cambridge Astrophys. Ser., 43, 8

Cao, Y., Kasliwal, M. M., Neill, J. D., et al. 2012, ApJ, 752, 133

Darnley, M. J., Bode, M. F., Kerins, E., et al. 2006, MNRAS, 369, 257

Darnley, M. J., Williams, S. C., Bode, M. F., et al. 2014, A\&A, 563, L9

Darnley, M. J., Henze, M., Steele, I. A., et al. 2015a, A\&A, 580, A45

Darnley, M. J., Henze, M., Shafter, A. W., Kato, M. 2015b, ATel, 7964

Disney, M. J., \& Wallace, P. T. 1982, QJRAS, 23, 485

Henze, M., Ness, J.-U., Darnley, M. J., et al. 2014, A\&A, 563, L8

Henze, M., Ness, J.-U., Darnley, M. J., et al. 2015, A\&A, 580, A46

Hubble, E. P. 1929, ApJ, 69, 103

Kato, M., Saio, H., Hachisu, I., \& Nomoto, K. 2014, ApJ, 793, 136

Kato, M., Saio, H., \& Hachisu, I. 2015, ApJ, 808, 52

Korotkiy, S., \& Elenin, L. 2010, CBAT, http://www.cbat.eps.harvard. edu/unconf/followups/J00452885+4154094.html

Massey, P., Olsen, K. A. G., Hodge, P. W., et al. 2006, AJ, 131, 2478

Nishiyama, K., \& Kabashima, F. 2008, CBAT, http://www.cbat.eps. harvard.edu/iau/CBAT_M31.html\#2008-12a

Nishiyama, K., \& Kabashima, F. 2012, CBAT, http://www.cbat.eps . harvard.edu/unconf/followups/J00452884+4154095.html

Pagnotta, A., \& Schaefer, B. E. 2014, ApJ, 788, 164

Pietsch, W., Haberl, F., Sala, G., et al. 2007, A\&A, 465, 375

Shafter, A. W., Hornoch, K., Ciardullo, J. V. R., Darnley, M. J., \& Bode, M. F. 2012, ATel, 4503

Shafter, A. W., Henze, M., Rector, T. A., et al. 2015, ApJS, 216, 34

Tang, S., Cao, Y., \& Kasliwal, M. M. 2013, ATel, 5607

Tang, S., Bildsten, L., Wolf, W. M., et al. 2014, ApJ, 786, 61

Williams, B. F., Garcia, M. R., Kong, A. K. H., et al. 2004, ApJ, 609, 735

Williams, S. C., Darnley, M. J., Bode, M. F., Keen, A., \& Shafter, A. W. 2014, ApJS, 213, 10

Woudt, P. A., \& Ribeiro, V. A. R. M. 2014, Stellar Novae: Past and Future Decades (San Francisco, CA: ASP), ASP Conf. Ser., 490

White, N. E., Giommi, P., Heise, J., Angelini, L., \& Fantasia, S. 1995, ApJ, 445, L125 